\title{
Rho Kinase Inhibition by Fasudil Has Anti-inflammatory Effects in Hypercholesterolemic Rats
}

\author{
Zhihong MA,${ }^{a}$ Jianping ZHANG, ${ }^{a}$ Rongpin Du, ${ }^{b}$ Ensheng $\mathrm{JI}_{\mathrm{I}}{ }^{a}$ and Li CHU ${ }^{*, a}$ \\ ${ }^{a}$ Department of Pharmacology, School of Basic Medicine, Hebei Medical University; No. 326, Xinshi South Road, \\ Shijiazhuang, Hebei 050091, Shijiazhuang, China: and ${ }^{b}$ Center of Cardiology, Hebei General Hospital; No. 348, Heping \\ West Road, Shijiazhuang, Hebei 050051, Shijiazhuang, China. \\ Received April 8, 2011; accepted August 3,2011; published online August 15, 2011
}

Inhibition of Rho kinase (ROCK) ameliorates many cardiovascular dysfunctions. The aim of the current study is to investigate the anti-inflammatory effects of fasudil, a selective ROCK inhibitor, on high cholesterol diet-induced hypercholesterolemic rats and its possible mechanisms. In hypercholesterolemic rats, we found the serum levels of total cholesterol (TC), low-density lipoprotein cholesterol (LDL-C) and several inflammatory markers including interleukin (IL)-8, IL-6, C-reaction protein (CRP) and soluble intercellular adhesion molecule (sICAM)-1 significantly elevated, while those of high-density lipoprotein cholesterol (HDL-C) and triglyceride (TG) decreased. Moreover, mRNA expressions of ROCK and nuclear factor-kappa B (NF- $\mathrm{B}$ ) and activity of ROCK in thoracic aorta were greatly up-regulated. Remarkably, administrating fasudil $(10,30 \mathrm{mg} / \mathrm{kg}$ per day) or simvastatin $(10 \mathrm{mg} / \mathrm{kg}$ per day) to hypercholesterolemic rats for 2 weeks, activation of ROCK and NF- $\kappa \mathrm{B}$ in thoracic aorta were suppressed, status of dyslipidemia were improved and inflammatory markers lowered. From the histopathological examination, fasudil treatment was found to lessen the thickening noted in the aortic intima and media of the hypercholesterolemic rats. These results suggested fasudil-induced inhibition of ROCK may improve lipid metabolism and has anti-inflammatory effect, which might expand the clinical application of fasudil as a new therapy for hypercholesterolemia and preventing the development of atherosclerosis.

Key words Rho kinase; fasudil; anti-inflammation; hypercholesterolemia; rat

Atherosclerosis is the leading cause of cardiovascular morbidity and mortality in the world, and hypercholesterolemia is one of the most important risk factors for atherosclerosis and subsequent cardiovascular disease. Various factors are known to be involved in the progression of hypercholesterolemia, including dyslipidemia, production of inflammatory cytokines and oxidative stress. Hypercholesterolemia-induced inflammatory response has been reported in rabbits within a few days of feeding a high cholesterol enriched diet, that is, long before the appearance of fatty streak lesions in large arteries. ${ }^{1)}$ Although the precise intracellular transduction pathways involved remain unknown, previous studies strongly indicated that nuclear factor-kappa B (NF- $\kappa \mathrm{B})$ was involved in this process. ${ }^{2)}$

Rho kinase (ROCK) is the first and the best-characterized effector of the small G-protein RhoA. Besides its effect on the regulation of actin cytoskeleton, ROCK plays a central role in diverse cellular functions such as smooth muscle cell contraction, stress fiber formation, and cell migration and proliferation. ${ }^{3)}$ Accumulating evidence indicates that ROCKmediated pathway is involved in all stages of the inflammatory process. ROCK has been shown to be activated at inflammatory arteriosclerotic lesions in human, ${ }^{4)}$ and inhibition of ROCK significantly limited early atherosclerotic plaque formation in apolipoprotein E-knockout (apoE-KO) mice ${ }^{5)}$ and low-density lipoprotein receptor knockout (LDLR-KO) mice $^{6)}$ by inhibiting NF- $\kappa \mathrm{B}$ activation. The anti-inflammatory properties of statins (3-hydroxy-methylglutaryl CoA reductase inhibitors) are believed to be mediated by inhibition of Rho protein isoprenylation and the subsequent activation of ROCK. ${ }^{7,8)}$ Recently, Yu et al. ${ }^{9)}$ reported that fasudil, a potent and specific inhibitor of ROCK, suppressed the inflammatory response in autoimmune encephalomyelitis by inhibiting T-cell proliferation and infiltrates of inflammatory cells within the central nervous system. Therefore, ROCK inhibitors might be a promising approach for the treatment of patients with hypercholesterolemia. Thus, the purpose of this study is to investigate the effect of fasudil, an inhibitor of ROCK, on high cholesterol diet (HCD)-induced hypercholesterolemic rats and its possible mechanisms in vivo.

\section{MATERIALS AND METHODS}

Materials Fasudil hydrochloride was obtained from Chase Sun Pharmaceutical Co., Ltd. (Tianjin, China). Unless otherwise stated, other chemical reagents were purchased from Sigma Chemical Co. (St. Louis, U.S.A.).

Animals Male Sprague-Dawley (SD) rats weighing 170 $210 \mathrm{~g}$ were purchased from Experimental Animal Center, Hebei Medical University, and they were housed in plastic cages with well ventilated stainless steel grid top at room temperature with a $12 \mathrm{~h}$ light/dark cycle, meanwhile, they received normal rat chow as well as drinking water ad libitum. All animal-handling procedures were in accordance with the Guidelines of Animal Experiments from the Committee of Medical Ethics, National Health Department of China.

Experimental Design After 1 week of accustomization, thirty male $\mathrm{SD}$ rats were weighed and randomized into five groups ( $n=6$ in each group): (1) Normal control group (Con): rats were fed a normal rat chow only. In addition to the normal control group, the other rats were all fed a highcholesterol diet (normal rat chow supplemented with 4\% cholesterol, $1 \%$ sodium tauroglycocholate and $0.5 \%$ propylthiouracil; HCD) for 4 weeks $^{10)}$ and received different treatment 2 weeks after the HCD feeding. (2) The high-cholesterol group (HCD): rats received physiological saline ( $1 \mathrm{ml} / 100 \mathrm{~g}$ per day) by intraperitoneal injection. (3) The simvastatin-treated group (Sim): rats were administered simvas- 
tatin (10 mg/kg per day) by oral gavage. (4) The low-dose fasudil treated group: rats received fasudil hydrochloride (10 mg/kg, F10) by intraperitoneal injection. (5) The highdose fasudil treated group: rats received fasudil hydrochloride $(30 \mathrm{mg} / \mathrm{kg}$ per day, F30) by intraperitoneal injection. Treatment lasted for 2 weeks, and the situations of food-intake and activities of all rats were observed carefully everyday.

On the 29th day of experiment, final body weight of each animal was recorded and overnight fasted animals were sacrificed by decapitation after sodium pentobarbital anesthesia. Blood from each animal was collected and serum was isolated for the estimation of lipid profiles and inflammatory markers. The aorta was immediately excised and rinsed in cold physiological saline. Just below the branch of the left subclavicular artery, the thoracic aorta of $3 \mathrm{~mm}$ length was immediately prepared for histological examination and immunohistochemical analysis. The remaining aortic samples were frozen and stored at $-86^{\circ} \mathrm{C}$ for reverse transcriptionpolymerase chain reaction (RT-PCR) and western blot analysis.

Estimation of Lipid Profiles in Serum Fasting serum lipids including total cholesterol (TC), triglyceride (TG), high-density lipoprotein cholesterol (HDL-C) and low-density lipoprotein cholesterol (LDL-C) were estimated by colorimetric methods with commercially available kits (Jian Cheng Biological Engineering Institute, Nanjing, China).

Measurement of Inflammatory Markers in Serum The concentrations of serum interleukin-6 (IL-6) and interleukin-8 (IL-8) were determined by radioimmunoassay (RIA) according to the instructions of the kits (Northern Institute of Biotechnology, Beijing, China), whereas those of soluble intercellular adhesion molecule (sICAM)-1 and C-reaction protein (CRP) were assessed by enzyme-linked immunosorbent assay (R\&D Systems, Minneapolis, U.S.A.).

RT-PCR Analysis for mRNA of ROCK1 and p65 Subunit of NF- $\boldsymbol{\kappa}$ B in Thoracic Aorta Total RNA was isolated from rapidly frozen aortic strips using Trizol reagent (Invitrogen, Carlsbad, U.S.A.) according to the manufacturer's instructions. The concentration and purity of total RNA were determined spectrophotometrically at wave lengths 260/280 nm. cDNA was synthesized using oligo(dT)20 and AMV reverse transcriptase (Takara Bio Inc., Dalian, China). PCR was performed by PCR kit (Takara Bio Inc., Dalian, China) for 30 cycles (ROCK1 and p65 subunit of NF- $\kappa$ B) or 25 cycles (glyceraldehyde-3-phosphate dehydrogenase $(\mathrm{GAPDH})$ ) at $94^{\circ} \mathrm{C}$ for $30 \mathrm{~s}, 55^{\circ} \mathrm{C}$ (ROCK1) or $60^{\circ} \mathrm{C}$ (GAPDH and p65 subunit of NF- $\kappa$ B) for $50 \mathrm{~s}, 72^{\circ} \mathrm{C}$ for $90 \mathrm{~s}$. PCR products were normalized based on the level of GAPDH. Primers were designed according to mRNA sequences published in GenBank. ${ }^{11,12)}$ Accession code for
ROCK1 is U61266, p65 subunit of NF- $\kappa \mathrm{B}$ is AF079314 and GAPDH is M17701 (Table 1).

Western Blot Analysis of ROCK Activity in Thoracic Aorta ROCK activity in aortic tissue was assayed as the amount of phosphorylated myosin phosphatase target subunit (p-MYPT1) at Ser-695 of myosin light chain (MLC) phosphatase.

Western blot analysis was performed as previously described, ${ }^{13)}$ with some modification. Briefly, strips of the aorta were homogenized in RIPA buffer (Sigma Chemical Co., St. Louis, U.S.A.). After the lysates were centrifuged at $12000 \mathrm{rpm}$ for $5 \mathrm{~min}$, supernatants were collected. Then the proteins were quantified by a modified Lowry protein assay. Equal amounts of proteins were separated by $12 \%$ sodium dodecyl sulfate-polyacrylamide gels electrophoresis (SDSPAGE), and electro-transferred to a polyvinylidene difluoride (PVDF) membrane, and finally detected by a specific polyclonal antibody (Santa Cruz Biotechnology Inc., Santa Cruz, U.S.A.), which recognizes MYPT1 phosphorylated at Ser695 by ROCK. GAPDH was used to monitor equal loading.

Immunohistochemical Analysis of NF- $K \mathrm{~B}$ in Thoracic Aorta The expression and localization of NF- $\kappa \mathrm{B}$ was determined by detection the p65 subunit of NF- $\kappa$ B with immunohistochemical analysis. An immunohistochemical procedure was applied with minor modification. ${ }^{14)}$

Endogenous peroxidase activity was inhibited by incubation with $0.3 \%$ hydrogen peroxide. After blocking sections with $5 \%(\mathrm{w} / \mathrm{v})$ bovine serum albumin (BSA), the sections were then treated with primary rabbit NF- $\kappa \mathrm{B}$ p 65 polyclonal antibodies (Santa Cruz Biotechnology Inc., Santa Cruz, U.S.A.) in phosphate buffered saline (PBS) containing $1 \%$ serum (overnight at $4{ }^{\circ} \mathrm{C}$ ), biotin-labeled secondary antibody (30 min at room temperature) and then avidin-biotin-peroxidase complex (Vector Laboratories, Burlingame, U.S.A.). The sections were stained using a diaminobenzidine (DAB) substrate kit (Vector Laboratories, Burlingame, U.S.A.) and the nuclei were counterstained with hematoxylin. The expression and localization of the p65 subunit of NF- $\kappa \mathrm{B}$ was determined by microscopic observation of the brown peroxidase reaction product on the vascular wall of the thoracic aorta.

Table 1. PCR Primer Sequences

\begin{tabular}{lll}
\hline \hline \multicolumn{2}{l}{ PCR primer } & \\
\hline \multirow{2}{*}{ ROCKI } & Sense & 5'-GAGCAACTACGATGTGCCTGA AAAG-3' \\
& Antisense & 5'-GAT GAC GTT TGA TTT CCT CTAC-3' \\
\multirow{2}{*}{ NF- $\kappa$ B } & Sense & 5'-ACG ATC TGT TTC CCC TCA TCT-3' \\
& Antisense & 5'-TGC TTC TCT CCC CAG GAATA-3' \\
GAPDH & Sense & 5'-CAT GGT CTA CAT GTT CCAGT-3' \\
& Antisense & 5'-GGC TAA GCA GTT GGT GGTGC-3'
\end{tabular}

Table 2. Effects of Fasudil on Serum Lipids (mmol/l, $n=6$ )

\begin{tabular}{|c|c|c|c|c|c|}
\hline Group & Con & $\mathrm{HCD}$ & Sim & F10 & F30 \\
\hline $\mathrm{TC}$ & $1.41 \pm 0.17$ & $12.17 \pm 1.10^{*}$ & $4.83 \pm 0.19^{*} \dagger$ & $8.13 \pm 1.43^{*, \dagger}$ & $7.72 \pm 1.16^{*, \dagger}$ \\
\hline TG & $0.88 \pm 0.11$ & $0.33 \pm 0.09 *$ & $0.32 \pm 0.10^{*}$ & $0.24 \pm 0.07 *$ & $0.23 \pm 0.04 *$ \\
\hline LDL & $0.91 \pm 0.08$ & $1.73 \pm 0.33^{*}$ & $1.18 \pm 0.06^{* \dagger \dagger}$ & $1.71 \pm 0.12 *$ & $1.66 \pm 0.12 *$ \\
\hline HDL & $2.31 \pm 0.47$ & $1.02 \pm 0.04 *$ & $1.47 \pm 0.37^{*, \dagger}$ & $1.21 \pm 0.12 *$ & $2.13 \pm 0.17^{\dagger}$ \\
\hline
\end{tabular}

Con: Control group; HCD: HCD group; Sim: simvastatin-treated group; F10: low-dose fasudil treated group; F30: high-dose fasudil treated group. Results are expressed as means \pm S.D. $* p<0.05$ versus control group. $\dagger p<0.05$ versus $\mathrm{HCD}$ group. 
Histological Examination for Thoracic Aorta The aortas isolated from each group were fixed in $10 \%(\mathrm{v} / \mathrm{v})$ formalin in $50 \mathrm{~mm}$ potassium phosphate buffer ( $\mathrm{pH} \mathrm{7.0)}$ for $24 \mathrm{~h}$ at $4{ }^{\circ} \mathrm{C}$. The tissues were subsequently embedded in paraffin, sectioned $(4 \mu \mathrm{m})$ and stained with hematoxylin and eosin (H-E). Slides were observed under optical microscope (Olympus Japan Co., Tokyo, Japan) at a magnification of $400 \times$ for histological changes.

Statistical Analysis Data analysis was conducted with SPSS software package for Windows (Version 11.5; SPSS Inc., Chicago, U.S.A.). Data distributions were examined for normality and homogeneity of variance. The values are expressed as means \pm S.D. if they are normally distributed. Comparisons among groups were performed using one-way analysis of variance (ANOVA) with post hoc test, followed by the least significance difference (LSD) test. For nonparametric analysis, we used the Mann-Whitney $U$-test with post $h o c$ analysis to evaluate the difference among 5 groups. A value of $p<0.05$ was accepted as statistically significant.

\section{RESULTS}

Effects of Fasudil on Serum Lipids The serum lipids were measured and presented in Table 2. HCD group had significantly higher levels of TC and LDL-C, as well as lower levels of TG and HDL-C compared with control group. In consistent with published data, simvastatin treatment resulted in marked decrease of TC and LDL-C, and increase of HDL$\mathrm{C}$ levels in serum compared with HCD group, but had no effect on TG level. High-dose $(30 \mathrm{mg} / \mathrm{kg})$ and low-dose $(10 \mathrm{mg} /$ $\mathrm{kg}$ ) of fasudil reduced TC level compared with HCD group, with the high-dose more effective than the low-dose. Highdose, but not low-dose fasudil, increased HDL-C compared to HCD group. Serum TG level tended to be lower after fasudil treatment, although not so apparently. Fasudil had no effect on serum LDL-C level.

Effects of Fasudil on Inflammatory Markers in Serum CRP, sICAM, IL-6 and IL-8 were tested to show the inflammatory markers in serum. As shown in Fig. 1, HCD group had significantly elevated concentrations of CRP, sICAM, IL6 and IL-8 compared with control group. Simvastatin re- sulted in significant suppression of CRP, sICAM, IL-6 and IL-8. Both doses of fasudil led to significant decreases of IL6, IL-8 and sICAM levels. However, only high-dose fasudil reduced CRP level.

Effects of Fasudil on ROCK Protein in Thoracic Aorta HCD group showed higher level of ROCK mRNA expression in the thoracic aorta than control group. However, the mRNA expression was reduced remarkably after treatment with simvastatin or both doses of fasudil (Fig. 2A).

In addition, western blot analysis for p-MYPT1 was performed to quantify the activity of ROCK in hypercholesterolemic aorta. The extent of p-MYPT1 was significantly elevated in the thoracic aorta of HCD group, which was dramatically suppressed by treatment with simvastatin or fasudil (Fig. 2B).

Effects of Fasudil on p65 Subunit of NF- $\kappa$ B mRNA and Protein Levels in Thoracic Aorta Immunohistochemical analysis showed relatively abundant expression of the NF- $\kappa \mathrm{B}$ immunoactivity in both the endothelial and smooth muscle cells of the thoracic aorta in the HCD group, as well as the localization in the nuclei of intimal endothelial cells and medial smooth muscle cells. However, NF- $\kappa$ B expression was
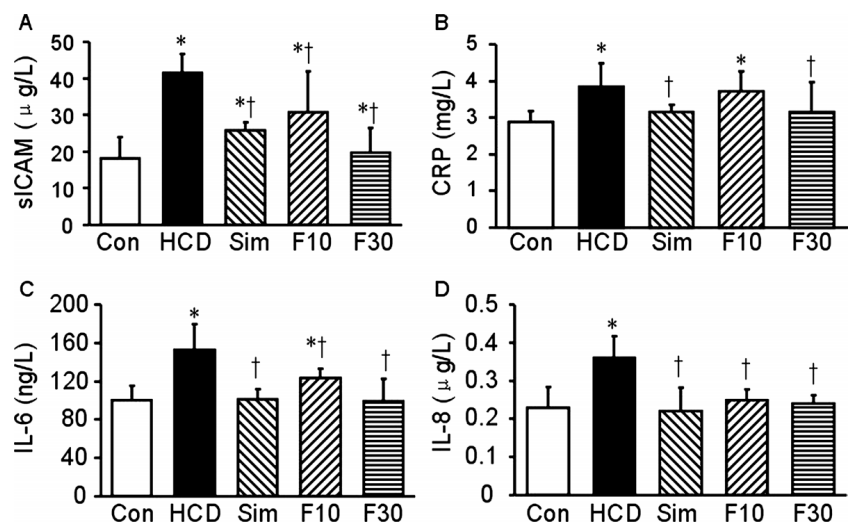

Fig. 1. Effect of Fasudil on Inflammatory Markers in Serum

Serum sICAM (A), CRP (B), IL-6 (C) and IL-8 (D) levels after treatment with physiological saline (HCD), low-dose fasudil (10 mg/kg, F10), high-dose fasudil (30 mg/kg, F30) or simvastatin $(10 \mathrm{mg} / \mathrm{kg}$, Sim). Values are expressed as means \pm S.D. $* p<0.05$ versus control group (Con). $\uparrow p<0.05$ versus $\mathrm{HCD}$ group. $n=6$ per group.
A
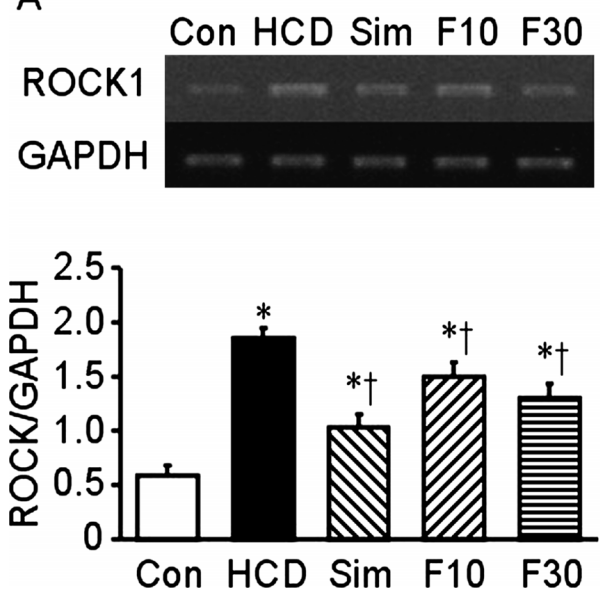

B
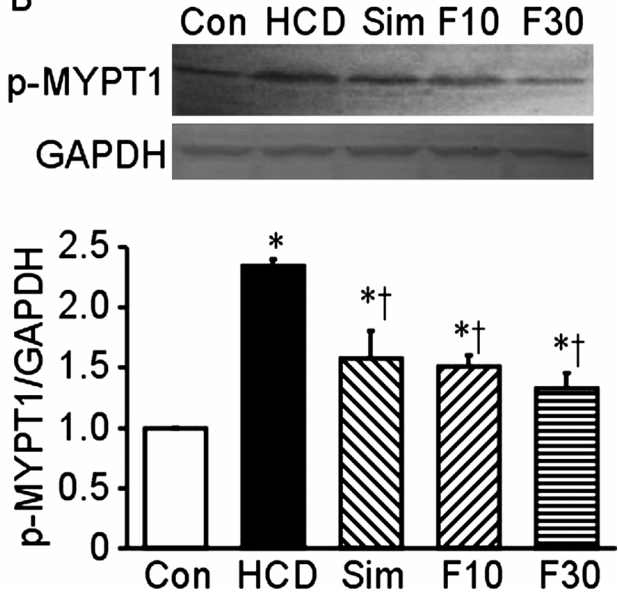

Fig. 2. Effect of Fasudil on ROCK in Thoracic Aorta

Expressions of ROCK mRNA (A) and p-MYPT1 protein (B) in thoracic aorta after treatment with physiological saline (HCD), simvastatin (10 mg/kg, Sim), low-dose fasudil $(10 \mathrm{mg} / \mathrm{kg}, \mathrm{F} 10)$ or high-dose fasudil ( $30 \mathrm{mg} / \mathrm{kg}, \mathrm{F} 30)$. Top panels are representative typical RT-PCR and Western blot bands. Bottom panels show percent of ROCK (A) and pMYPT1 (B) in relation to GAPDH. Values are expressed as means \pm S.D. $* p<0.05$ versus control group (Con). ${ }^{\dagger} p<0.05$ versus $\mathrm{HCD}$ group. $n=6$ per group. 

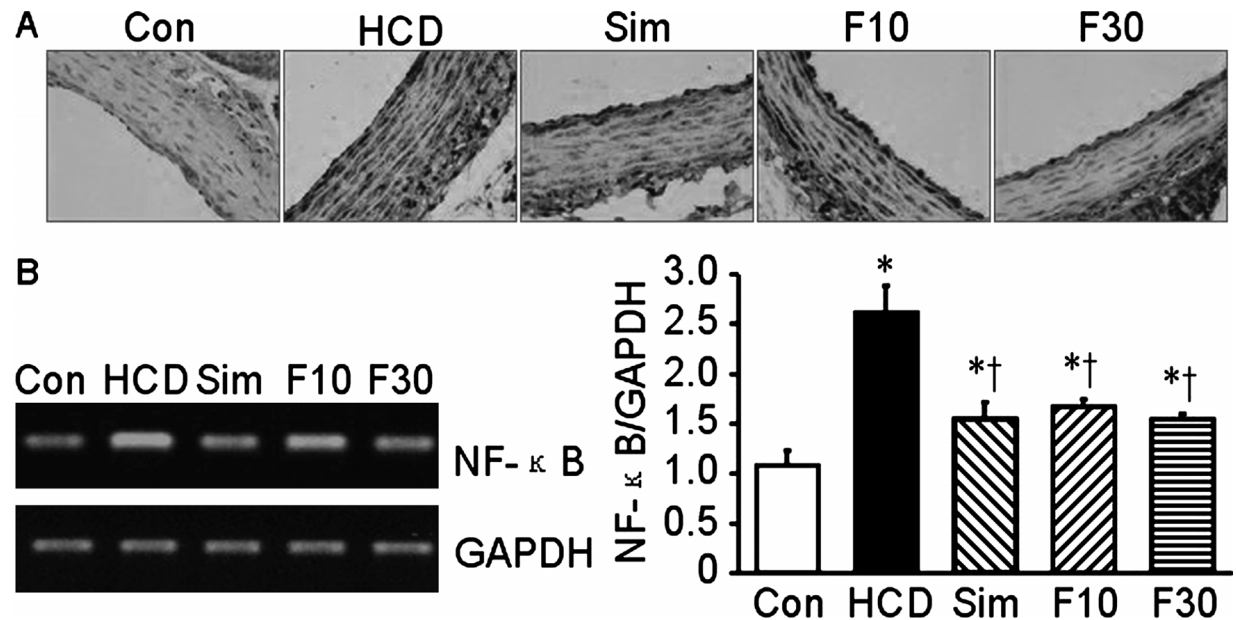

Fig. 3. Effect of Fasudil on NF- $\kappa$ B in Thoracic Aorta

(A) Immunohistochemical staining for p65 subunit of NF- $\kappa \mathrm{B}$ in the thoracic aorta (magnification, $400 \times$ ). Thoracic aorta obtained from Control group (Con), HCD group (HCD), simvastatin-treated group (Sim), low-dose fasudil treated group (F10), and high-dose fasudil treated group (F30). (B) p65 subunit of NF- $\kappa$ B mRNA. Left panel is representative of typical RT-PCR bands; right panel shows percent of NF- $\kappa \mathrm{B}$ in relation to GAPDH. Values are expressed as means \pm S.D. $* p<0.05$ versus control group. $\dagger p<0.05$ versus HCD group. $n=6$ per group.
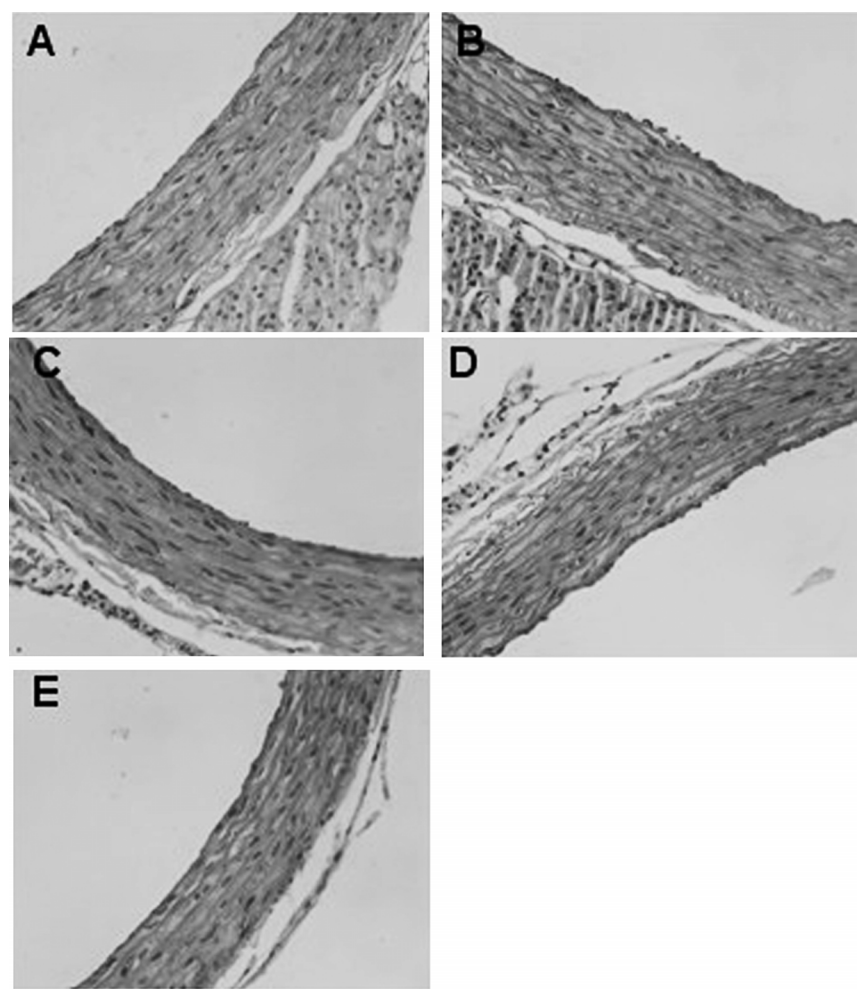

Fig. 4. Effect of Fasudil on Histopathological Changes of Thoracic Aorta Representative microscopic photographs of thoracic aorta stained with Hematoxylin and Eosin (magnification $\times 400$ ). Thoracic aorta obtained from Control group (A) HCD group (B), simvastatin-treated group (C), low-dose fasudil treated group (D), and high-dose fasudil treated group (E).

not found in the nuclei of vascular cells of control rats. After treatment with simvastatin or fasudil, the expression and localization of NF- $\kappa \mathrm{B}$ in nuclei significantly declined (Fig. 3A).

The mRNA expression of $p 65$ subunit of NF- $\kappa$ B in HCD group was higher than that in control group. Simvastatin and fasudil groups showed remarkably lower p65 subunit of NF$\kappa \mathrm{B}$ mRNA expressions compared with HCD group (Fig. 3B).
Histological Examination for Thoracic Aorta Figure 4 shows the H-E stained aortic segments from the experimental groups. In the aortic segments from the HCD group, there was a pathological thickening of the intima and media. This change was ameliorated by simvastatin and both doses of fasudil treatments.

\section{DISCUSSION}

Hydroxyfasudil is a highly selective ROCK inhibitor. ${ }^{15,16)}$ In this study, we used a previously published dose $(30 \mathrm{mg} / \mathrm{kg}$ daily) ${ }^{17)}$ that resulted in a marked inhibition of in vivo ROCK activity as shown in HCD-induced hypercholesterolemic aortas of fasudil-treated rats. This inhibition of ROCK activity was associated with improving lipid metabolism and anti-inflammation in vivo. Simvastatin was used as a standard agent in the present study.

Rats fed with a diet supplemented with $4 \%$ cholesterol, $1 \%$ sodium tauroglycocholate and $0.5 \%$ propylthiouracil for 4 weeks served as the experimental model of early phase of atherogenesis. The results of the present work revealed these animals developed obvious dyslipidemia, with increasing of $\mathrm{TC}$ and LDL-C, and decreasing of TG and HDL-C. The changes of TC, LDL-C and HDL-C were consistent with other studies, whereas, that of TG was different from them. ${ }^{10,18)}$ In line with other published studies, simvastatin improved on serum lipid parameters. Moreover, treatment with fasudil markedly decreased the serum level of TC and elevated the serum level of HDL-C. Shah and Singh ${ }^{19)}$ also reported that fasudil markedly decreased serum concentrations of TC and TG; and increased that of HDL-C in hypercholesterolemic rats. The observed effect of fasudil on improve lipid metabolism might result from the activating peroxisome proliferator-activated receptor (PPAR)- $\alpha$, which, secondarily mediated increase in production and secretion of apoA- $\mathrm{I}^{20,21)}$ and decrease in the synthesis of cholesterol ${ }^{22)}$ by inhibition of ROCK. Noticeably, neither simvastatin nor fasudil could completely reverse the studied lipids to normal levels. This could be the result of persistent HCD feeding and suggested that dietary control should be the most impor- 
tant strategy in treating hyperlipemia.

Accumulating evidence shows that early atherosclerosis is characterized by the attraction of monocytes to the endothelium and penetration into the subendothelial space. Enhanced expressions of pro-inflammatory cytokines like ICAM, IL-8 and IL- 6 accelerate this process. IL-6 also stimulates the production of CRP by hepatocytes. ${ }^{23,24)}$ In addition, the levels of cytokines were visibly increasing in serum and circulating blood leukocytes in hypercholesterolemic patients compared with healthy subjects. ${ }^{25}$ In this study, we detected significant amount of serum CRP, sICAM, IL-8, and IL-6 in hypercholesterolemic rats compared with control rats. We also demonstrated that simvastatin treatment could suppress the expression of CRP, sICAM, IL-6, and IL-8 in the serum. More notably, our results suggested that both doses of fasudil significantly ameliorated the elevation of sICAM, IL-6, and IL-8 levels, whereas, only high-dose fasudil restrained the CRP level. These data, together with previous studies showing anti-inflammation of statins are believed to be mediated by inhibition of ROCK pathway, ${ }^{7,8)}$ indicated down-regulating the production of pro-inflammatory cytokines in fasudiltreated hypercholesterolemic rats might be through inhibiting ROCK activity.

The NF- $\kappa \mathrm{B}$ is an important mediator in the vascular inflammatory processes, and its activation results in the up-regulation of inflammatory and cell adhesion molecules. Studies have shown NF- $\kappa \mathrm{B}$ activation in advanced human atherosclerotic lesions ${ }^{26}$ and early atherosclerotic plaques in LDLR$\mathrm{KO}$ mice. ${ }^{6}$ Furthermore, studies in vitro suggest that statins inhibit the activation of NF- $\kappa \mathrm{B} .{ }^{27,28)}$ In this study, we showed that the mRNA expression of p65 subunit of NF- $\kappa$ B was upregulated, and translocation of activated NF- $\kappa \mathrm{B}$ from cytoplasm to nucleolus augmented in hypercholesterolemic rats. Our data of fasudil inhibited the expression and activation of $\mathrm{NF}-\kappa \mathrm{B}$ in thoracic aorta indicated a potentially important role for NF- $\kappa \mathrm{B}$ modulation in this context. RhoA was reported to activate NF- $\kappa \mathrm{B}$ in murine NIH-3T3 fibroblasts and simian COS-7 fibroblast-like cells. ${ }^{29,30)}$ Recently, fasudil was shown to restrain cytokine expressions in synoviocytes from rheumatoid arthritis patients and human endothelial cells by inhibiting NF- $\kappa \mathrm{B}$ activation. ${ }^{31)}$ Our observations that fasudil inhibited the expression and activation of NF- $\kappa \mathrm{B}$ in thoracic aorta indicated a potentially important role for NF- $\kappa \mathrm{B}$ modulation in this context. Although changes in p65 expression did not reflect NF- $\kappa \mathrm{B}$ activation, the pattern of p65 staining within the aortic wall highly suggested a nuclear associated staining. However, the precise mechanism of NF- $\kappa \mathrm{B}$ activation warrants further investigation.

The expression of Rho-kinase is accelerated by remnant lipoproteins $^{32)}$ or inflammatory stimuli through $\mathrm{PKC} / \mathrm{NF}-\kappa \mathrm{B}$ pathway ${ }^{33)}$ and positively involved in its own expression. ${ }^{34)}$ In the present study, significant up-regulation of ROCK1 mRNA expression was shown in hypercholesterolemic aorta. Treatment with simvastatin or fasudil obviously inhibited the ROCK-1 mRNA expression induced by hypercholesterolemia. These results indicate that hypercholesterolemia not only activates but also up-regulates ROCK-1 mRNA expression; we speculate that this may result from the activation of $\mathrm{NF}-\kappa \mathrm{B}$. Fasudil or simvastatin inhibits $\mathrm{NF}-\kappa \mathrm{B}$ translocation to the nucleus and next the activation by the inhibition of ROCK activity, which may account for the fact that they inhibits ROCK-1 mRNA expression. Of course, further studies will have to investigate the precise mechanism.

In accordance with the elevated inflammatory markers in serum from the HCD rats, histological examinations revealed a thickening of aortic intima and media that is compatible with the processes of atherosclerosis, while this morphological change could be prevented by treatment with simvastatin and fasudil. This finding further suggests anti-inflammatory effects of fasudil.

The effects of fasudil obtained at doses may be mediated through specific inhibition of ROCK. It has been demonstrated that hydroxyfasudil, a major active metabolite of fasudil after oral administration, has a more specific inhibitory effect on ROCK. The $\mathrm{IC}_{50}$ values of hydroxyfasudil for ROCK1 were $0.73 \mu \mathrm{mol} / 1,37 \mu \mathrm{mol} / 1$ for protein kinase A (PKA), and $>100 \mu \mathrm{mol} / 1$ for protein kinase $\mathrm{C}$ (PKC) $\alpha$, respectively. ${ }^{35)}$ Nonetheless, we cannot exclude the possibility that fasudil might inhibit other kinases.

In conclusion, the present study demonstrates that upregulated ROCK activity involves in inflammatory response in hypercholesterolemic rats, possibly through its modulatory activity on NF- $\kappa \mathrm{B}$ activation. Furthermore, it indicates that fasudil has anti-inflammatory effects and may improve dyslipidemia, at least in part, through inhibiting ROCK activity in the hypercholesterolemic rats. Taken together, our results implicate the therapeutic potential of fasudil for treating inflammation and hypercholesterolemia.

Acknowledgement We would like to thank Gang Cao, Huazhou Xu (Hebei Medical University, China) for technical assistance and Jianzhao Shen (Medtronic, Inc., U.S.A.), Hong Chang (Hebei Medical University, China) and Yue Wang (Nankai University, China) for linguistic support. This work was supported by the Natural Science Foundation of Hebei Province (C2007000804) and the Key Project of the Science and Technology Pillar Program of Tianjin (09JCYBJC10800), China.

\section{REFERNCES}

1) Scalia R., Appel J. Z. 3rd, Lefer A. M., Arterioscler. Thromb. Vasc. Biol., 18, 1093-1100 (1998)

2) Hajra L., Evans A. I., Chen M., Hyduk S. J., Collins T., Cybulsky M. I., Proc. Natl. Acad. Sci. U.S.A., 97, 9052-9057 (2000).

3) Riento K., Ridley A. J., Nat. Rev. Mol. Cell Biol., 4, 446- 456 (2003).

4) Nohria A., Prsic A., Liu P. Y., Okamoto R., Creager M. A., Selwyn A., Liao J. K., Ganz P., Atherosclerosis, 205, 517-521 (2009).

5) Wu D. J., Xu J. Z., Wu Y. J., Jean-Charles L., Xiao B., Gao P. J., Zhu D. L., Atherosclerosis, 207, 68-73 (2009).

6) Mallat Z., Gojova A., Sauzeau V., Brun V., Silvestre J.-S., Esposito B., Merval R., Groux H., Loirand G., Tedgui A., Circ. Res., 93, 884-888 (2003).

7) Ni W., Egashira K., Kataoka C., Kitamoto S., Koyanagi M., Inoue S., Takeshita A., Circ. Res., 89, 415-421 (2001).

8) Takemoto M., Liao J. K., Arterioscler. Thromb. Vasc. Biol., 21, 1712 1719 (2001).

9) Yu J. H., Ding J., Ma C. G., Sun C. H., Sun Y. F., Lu C. Z. N., Xiao B. G., J. Neurosci. Res., 88, 1664-1672 (2010).

10) Deepa P. R., Varalakshmi P., Clin. Chim. Acta, 339, 105-115 (2004).

11) Leung T., Chen X. Q., Manser E., Lim L., Mol. Cell. Biol., 16, 53135327 (1996).

12) Tso J. Y., Sun X. H., Kao T. H., Reece K. S., Wu R., Nucleic Acids Res., 13, 2485-2502 (1985).

13) Kimura K., Ito M., Amano M., Chihara K., Fukata Y., Nakafuku M., Yamamori B., Feng J., Nakano T., Okawa K., Iwamatsu A., Kaibuchi 
K., Science, 273, 245-248 (1996).

14) Kaltschmidt C., Kaltschmidt B., Henkel T., Stockinger H., Baeuerle P. A., Biol. Chem. Hoppe Seyler, 376, 9-16 (1995).

15) Higashi M., Shimokawa H., Hattori T., Hiroki J., Mukai Y., Morikawa K., Ichiki T., Takahashi S., Takeshita A., Circ. Res., 93, 767-775 (2003).

16) Shimokawa H., Seto M., Katsumata N., Amano M., Kozai T., Yamawaki T., Kuwata K., Kandabashi T., Egashira K., Ikegaki I., Asano T., Kaibuchi K., Takeshita A., Cardiovasc. Res., 43, 1029-1039 (1999).

17) Li F. H., Xia W., Li A. W., Zhao C. F., Sun R. P., Pharmacol. Res., 55, 64-71 (2007).

18) Akila M., Devaraj H., Vascul. Pharmacol., 49, 173-177 (2008).

19) Shah D. I., Singh M., Can. J. Physiol. Pharmacol., 84, 835-845 (2006).

20) Noguchi M., Hosoda K., Fujikura J., Fujimoto M., Iwakura H., Tomita T., Ishii T., Arai N., Hirata M., Ebihara K., Masuzaki H., Itoh H., Narumiya S., Nakao K., J. Biol. Chem., 282, 29574-29583 (2007).

21) Martin G., Duez H., Blanquart C., Berezowski V., Poulain P., Fruchart J. C., Najib-Fruchart J., Glineur C., Staels B., J. Clin. Invest., 107, 1423-1432 (2001).

22) Knight B. L., Patel D. D., Humphreys S. M., Wiggins D., Gibbons G. F., J. Lipid Res., 44, 2049-2058 (2003).

23) Libby P., Nature (London), 420, 868 -874 (2002).

24) Nohria A., Grunert M. E., Rikitake Y., Noma K., Prsic A., Ganz P.,
Liao J. K., Creager M. A., Circ. Res., 99, 1426-1432 (2006).

25) Ito T., Ikeda U., Curr. Drug Targets, 2, 257-265 (2003).

26) Kowalski J., Okopien B., Madej A., Makowiecka K., Zielinski M., Kalina Z., Herman Z. S., Int. J. Clin. Pharmacol. Ther, 39, 48-52 (2001).

27) Brand K., Page S., Rogler G., Bartsch A., Brandl R., Knuechel R., Page M., Kaltschmidt C., Baeuerle P. A., Neumeier D., J. Clin. Invest. 97, 1715-1722 (1996).

28) Wang J. Y., Tokoro T., Higa S., Kitajima I., Biol. Pharm. Bull., 29 , 634-639 (2006).

29) Ortego M., Bustos C., Hernandez-Presa M. A., Tunon J., Diaz C., Hernandez G., Egido J., Atherosclerosis, 47, 253-261 (1999).

30) Perona R., Montaner S., Saniger L., Sánchez-Pérez I., Bravo R., Lacal J. C., Genes Dev., 11, 463 - 475 (1997).

31) Okamoto H., Yoshio T., Kaneko H., Yamanaka H., Arthritis Rheum., 62, 82-92 (2010).

32) Oi K., Shimokawa H., Hiroki J., Uwatoku T., Abe K., Matsumoto Y. Nakajima Y., Nakajima K., Takeichi S., Takeshita A., Arterioscler. Thromb. Vasc. Biol., 24, 918-922 (2004).

33) Hiroki J., Shimokawa H., Higashi M., Morikawa K., Kandabashi T., Kawamura N., Kubota T., Ichiki T., Amano M., Kaibuchi K., Takeshita A., J. Mol. Cell. Cardiol., 37, 537-546 (2004).

34) Shimokawa H., Jpn. Circ. J., 64, 1-12 (2000).

35) Rikitake Y., Kim H. H., Huang Z. H., Seto M., Yano K., Asano T., Moskowitz M. A., Liao J. K., Stroke, 36, 2251-2257 (2005). 